\title{
Beiträge zur Kenntnis des Vitamingehaltes von Belebtschlamm aus Abwasserreinigungsanlagen
}

\author{
Von V. Kocher und Ulrich A. Corti
}

(Mitteilung aus dem Mikrobiologischen Laboratorium an der Organisch-Chemischen Anstalt der Universität Basel und der Eidgenössischen Anstalt für Wasserversorgung, Abwasserreinigung und Gewässerschutz [EAWAG.] an der Eidgenössischen

Technischen Hochschule, Zürich)

Eingegangen am 22. Februar 1952

In Verbindung mit Untersuchungen, welche nähere Aufschlüsse über die Eigenschaften und insbesondere die Wirkungsweise des bei modernen Abwasserreinigungsanlagen eine fundamentale Rolle spielenden Belebtschlammes (boue activée, activated sludge) liefern sollen, wurden in demselben einige Vitamine bestimmt.

Als Material diente Belebtschlamm aus der technisch-biologischen Versuchsanlage der EAWAG. im Werdhölzli, der im mechanisch vorgereinigten Abwasser der Stadt Zürich erzeugt worden war.

Frischer Belebtschlamm vom September 195I wurde zentrifugiert (Wassergehalt des Zentrifugates im Mittel aus drei Einzelbestimmungen $9 \mathbf{I}, 2 \%)$ und das stichfeste Gut $\left(B_{\mathrm{I}}\right)$ tel quel untersucht.

Ferner wurde frischer Belebtschlamm vom August 195I zentrifugiert, bei $\operatorname{IO}^{0} \mathrm{C}$ getrocknet und fein vermahlen. Diese Trockensubstanz $\left(B_{\mathrm{II}}\right)$ wurde ebenfalls auf Vitamingehalt geprifft.

Die beiden mikrobiologisch analysierten Proben von Belebtschlamm enthalten nicht unbeträchtliche Mengen sogenannter «Schwebestoffe», das heisst Stoffe, welche in der der biologischen Abwasserreinigungsstufe vorgeschalteten mechanischen Kläranlage (Absetzbecken) nicht sedimentierten. Der Belebtschlamm besteht ausserdem aus Bakterien, Protozoen undsoweiter.

In der Tabelle I sind unsere Untersuchungsbefunde zusammengestellt. Die Unterschiede zwischen den einander entsprechenden Werten in den Kolonnen für $B_{\mathrm{I}}$ und $B_{\mathrm{II}}$ dürften sich, wenigstens zum Teil, durch die 
Verschiedenheit der Zusammensetzung der beiden zu verschiedenen Zeiten erhobenen Belebtschlammproben und die beim Trocknen der Probe $B_{I I}$ aufgetretenen Vitaminverluste (bakterielle Zersetzungen, Thermolabilität einzelner Vitamine) erklären lassen.

Tabelle 1

Vitamingebalt pon Belebtschlamm, berechnet auf $100 \mathrm{~g}$ Trockensubstan:

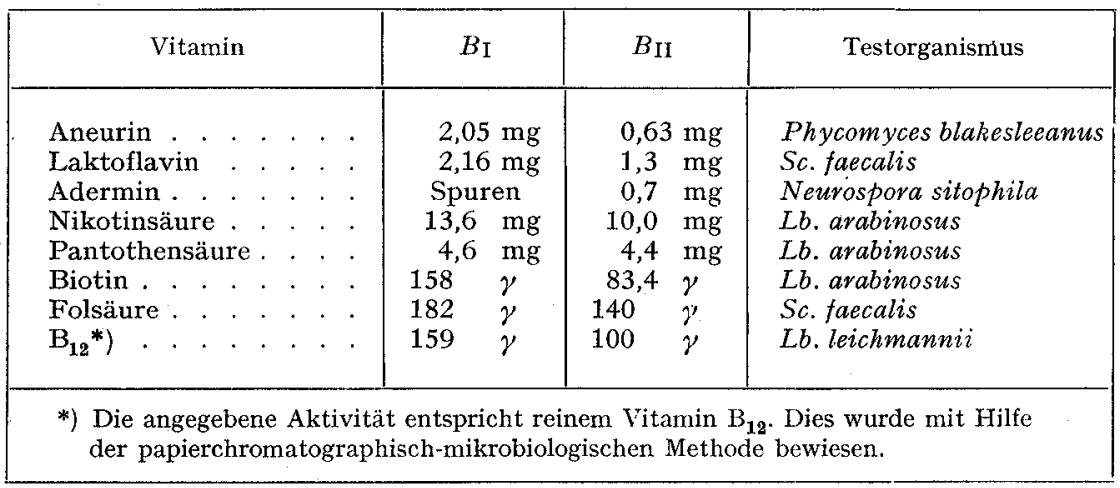

Ein Vergleich unserer Befunde mit dem Vitamingehalt anderer Substrate zeigt, dass die im Belebtschlamm vorkommenden Vitaminmengen nicht zu unterschätzen sind. So enthalten:

\begin{tabular}{|c|c|c|c|c|}
\hline $1000 \mathrm{~g}$ & Kuhmilch & $0,2-0,4$ & $\mathrm{mg}$ & Aneurin \\
\hline $1000 \mathrm{~g}$ & Kuhmilch & $1,0-2,5$ & $\mathrm{mg}$ & Laktoflavin \\
\hline $1000 \mathrm{~g}$ & Kuhmilch & $1,0-3,0$ & $\mathrm{mg}$ & Adermin \\
\hline $1000 \mathrm{~g}$ & Kuhmilch & $1,0-4,0$ & $\mathrm{mg}$ & Pantothensäure \\
\hline $1000 \mathrm{~g}$ & Kuhmilch & 0,05 & $\mathrm{mg}$ & Biotin \\
\hline $100 \mathrm{~g}$ & Trockenmilch & $0,1-0,4$ & $\mathrm{mg}$ & Nikotinsäureamic \\
\hline $100 \mathrm{~g}$ & Hefenextrakt & $0,1-0,35$ & $\mathrm{mg}$ & Folsäure \\
\hline $100 \mathrm{~g}$ & Rindsleber, getrocknet & 47 & $\gamma$ & $\left.B_{12}{ }^{2}\right)$ \\
\hline $100 \mathrm{~g}$ & Forellenleber, getrocknet & 42 & $\gamma$ & $\mathrm{B}_{12}^{2} \mathrm{2}$ \\
\hline
\end{tabular}

In der mechanischen Kläranlage der Stadt Zürich betrug der mittlere Abwasserzufluss bis vor kurzem zirka $2 \mathrm{~m}^{3} / \mathrm{s}$. Würde dieses Abwasser mechanisch vorgeklärt und anschliessend daran mittels eines Belebtschlammverfahrens einer sogenannten Teilreinigung unterzogen, analog wie dies im August und September I95I in der technischen Versuchsanlage der EAWAG. der Fall war, so würden pro $\mathrm{I}^{3}$ Abwasser zirka Ioo $\mathrm{g}$ Belebtschlamm-Trockensubstanz produziert werden. Rechnet man

1) H. T. Thompson, L. S. Dietrich, C. A. Elvehjem, J. Biol. Chem. 184, 175 (1950).

2) U. A. Corti und V. Kocher, Schweiz. Z. Hydrol. 13, 29 (1951). 
darnach mit einem Tagesanfall von $\mathrm{I}_{2} 800 \mathrm{~m}^{3}$ Abwasser, entsprechend $63,07 \cdot 10^{6} \mathrm{~m}^{3}$ pro Jahr, so würden sich auf Grund unserer Analysendaten die in der Tabelle 2 angeführten Werte als pro Jahr im erzeugten Belebtschlamm (Überschussschlamm $\sim 6307 \mathrm{t}$ Trockensubstanz) enthaltene Vitaminmengen ergeben.

Tabelle 2

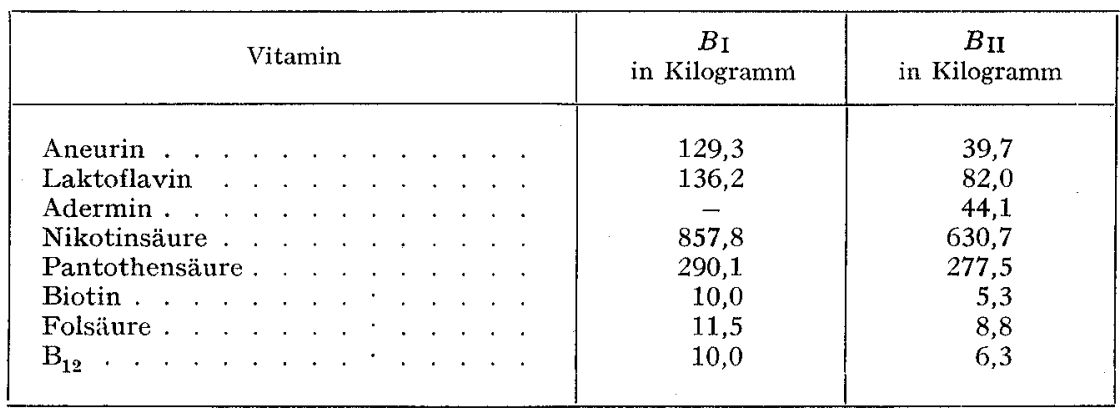

Besondere Beachtung scheint uns der relativ hohe Gehalt des Belebtschlammes an Vitamin $\mathrm{B}_{12}$ (Antiperniziosafaktor) $\mathrm{zu}$ verdienen. Die 6,3 bzw. Io,o kg $B_{12}$ entsprechen $\sim \mathrm{I}_{3} 420$ bzw. $\sim 2 \mathrm{I} 340 \mathrm{t}$ getrockneter Rindsleber ${ }^{1}$ ).

Man wird die Frage prüfen müssen, ob sich einwandfrei desinfizierter Belebtschlamm nicht eventuell als vitaminhaltiger Zusatz zu Tierfutter verwenden liesse. In diesem Zusammenhange erschiene es wünschenswert, zum Beispiel auch noch den Gehalt des Belebtschlammes an den fettlöslichen Vitaminen A (bzw. Karotin), D, E und K zu ermitteln.

\section{SUMMAR Y}

The vitamin content of activated sludge (pilot activated sludge plant treating predominant domestic sewage) has been measured microbiologically. Relatively high amounts of thiamin, riboflavin, pyridoxine, nicotinic acid, pantothenic acid, biotin, folic acid and vitamin $\mathrm{B}_{12}$ were found. $\mathrm{B}_{\mathbf{1 2}}$ was present in especially outstanding quantities.

1) Als die vorliegende Mitteilung bereits abgeschlossen war, erhielten wir Kenntnis der Publikation von S. R. Hoover und Mitarbeitern, Activated sludge as a source of vitamin $B_{12}$ for animal jeeds, Sewage and Industr. Wastes 24, 38-44 (1952). Die Verfasser fanden in getrocknetem Handelsdünger aus Belebtschlamm ca. 3,5-4 $\mathrm{mg} \mathrm{B}_{\mathbf{1 2}}$ pro Kilo. Leider ist uns die vorläufige Notiz von Hoover, L. JASEwicz und N. Porges, Vitamin $B_{12}$ in Activated Seqrage Sludge, Science 114, 213 (1951), entgangen. 